\title{
Intrinsic Activation of $\beta$-catenin Signaling by CRISPR/Cas9-mediated Exon Skipping Contributes to Immune Evasion in Hepatocellular Carcinoma
}

\section{Masafumi Akasu}

Tokyo Medical and Dental University

\section{Shu Shimada}

Tokyo Medical and Dental University

Ayano Kabashima

Tokyo Medical and Dental University

Yoshimitsu Akiyama

Tokyo Medical and Dental University

Masahiro Shimokawa

Tokyo Medical and Dental University

Keiichi Akahoshi

Tokyo Medical and Dental University

Atsushi Kudo

Tokyo Medical and Dental University

Shoji Yamaoka

Tokyo Medical and Dental University

Minoru Tanabe

Tokyo Medical and Dental University

Shinji Tanaka ( $\square$ tanaka.monc@tmd.ac.jp)

Tokyo Medical and Dental University

\section{Research Article}

Keywords: hepatocellular carcinoma (HCC), CTNNB1 , immunosuppressive tumor microenvironment, nuclear translocation

Posted Date: March 10th, 2021

DOl: https://doi.org/10.21203/rs.3.rs-263319/v1

License: (c) (1) This work is licensed under a Creative Commons Attribution 4.0 International License. Read Full License 


\section{Abstract}

Comprehensive analysis of clinical samples has recently identified molecular and immunological classification of hepatocellular carcinoma (HCC), and the CTNNB1 ( $\beta$-catenin)-mutated subtype exhibits distinctive characteristics of immunosuppressive tumor microenvironment. For clarifying the molecular mechanisms, we first established human and mouse HCC cells with exon 3 skipping of $\beta$-catenin, which promoted nuclear translocation and activated the Wnt/ $\beta$-catenin signaling pathway, by using newly developed multiplex CRISPR/Cas9-based genome engineering system. Gene set enrichment analysis indicated downregulation of immune-associated gene sets in the HCC cells with activated $\beta$-catenin signaling. T cell killing assays demonstrated that the mouse $C t n n b 7^{\triangle \mathrm{ex} 3} \mathrm{HCC}$ cells evaded immune surveillance. Comparative analysis of gene expression profiles between HCC cells harboring wild-type and exon 3 skipping $\beta$-catenin elucidated that the expression levels of eight cytokines were commonly decreased in human and mouse $\beta$-catenin-mutated HCC cells. Public exome and transcriptome data of 373 human HCC samples showed significant downregulation of five candidate cytokine genes, CCL20, CXCL1, CXCL2, NAMPT and VEGFA, in HCC tumors with $\beta$-catenin hotspot mutations. Taken together, this study discovered that cytokine controlled by $\beta$-catenin signaling activation could contribute to immune evasion, and provided novel insights into cancer immunotherapy for the $\beta$-catenin-mutated HCC subtype.

\section{Introduction}

Hepatocellular carcinoma (HCC) is a complex disease with various risk factors, that is, chronic infection with hepatitis $B$ virus and hepatitis $C$ virus, alcohol abuse, metabolic disease including obesity and diabetes, and dietary toxins such as aflatoxins and aristolochic acid ${ }^{1}$. Although anti-angiogenic agents and immune checkpoint blockers have currently emerged for HCC treatment ${ }^{2}$, it remains a leading cause of cancer-related death in the world ${ }^{3}$. To improve patient prognosis, categorization of tumor samples into subtypes and customization of cancer therapy for each subtype are essential in HCC, similarly to other types of cancer ${ }^{4}$. Several laboratories have proposed molecular classification of HCC on the basis of gene expression profiles in the past two decades, and a two-group model (proliferation and nonproliferation) is now widely accepted ${ }^{5,6}$. In our latest paper, recent advances in next generation sequencing technology elucidate that the non-proliferation group is further divided into two distinct subtypes, namely CTNNB1-mutated and metabolic disease-associated subtypes ${ }^{7}$.

Somatic mutations of $C T N N B 1$, encoding $\beta$-catenin, are most frequently identified in $\mathrm{HCC}$, and accumulated in exon 3 (amino acid position 5-80) corresponding to the serine/threonine (Ser/Thr) phosphorylation site for GSK3 $\beta$ which normally promotes ubiquitination and degradation of $\beta$-catenin. Gain-of-function mutations in exon 3 or exon 3 skipping events contribute to stabilization, translocation from cytoplasm to nucleus, and then activation of the Wnt/ $\beta$-catenin signaling pathway ${ }^{8,9}$. For examining this biological process, two genetically engineered mouse models have been developed; one is a transgenic mouse model with ectopic expression of mutated and stabilized $\beta$-catenin in which Ser33, Ser37, Thr41, and Ser45 are substituted by alanine residues ${ }^{10}$ or $\mathrm{N}$-terminal deletion ${ }^{11}$; the other is a 
Cre/ loxP-based mouse model harboring a mutant Ctnnb1 allele with loxP sequences in intron 2 and intron 3 for intrinsically skipping of exon $3^{12}$.

A series of studies has linked tumor-intrinsic Wnt/ $\beta$-catenin signaling not only to oncogenesis and stemness, but also to cancer immune surveillance. T cell-inflamed phenotype, characterized by CD $8+T$ cell infiltration, is closely correlated with the efficacy of immune checkpoint blockade, whereas non-T cellinflamed tumors rarely benefit. Luke et al. have recently addressed that the $\mathrm{Wnt} / \beta$-catenin signaling pathway is activated, particularly by CTNNB1 mutation, in non-T cell-inflamed tumors across cancer types including $\mathrm{HCC}^{13}$. In melanoma, $\beta$-catenin signaling upregulates IL-10 secretion, which impairs the capacity of dendritic cells (DCs) to cross-prime CD $8+$ cytotoxic T cells ${ }^{14}$, or downregulates CCL4 expression, resulting in $\mathrm{DC}$ defective recruitment and $\mathrm{T}$ cell exclusion ${ }^{15}$. However, although we and others have reported that $C T N N B 1$-mutated $\mathrm{HCC}$ shows immune suppression ${ }^{7,16}$, the molecular mechanism is not fully clarified in $\mathrm{HCC}^{17}$.

In this study, we established a novel model of intrinsically active $\beta$-catenin signaling by CRISPR/Cas9mediated exon skipping in human and mouse HCC cells, and investigated how tumor $\beta$-catenin signaling evades the immune system in HCC.

\section{Results}

\section{Exon 3 skipping of $\beta$-catenin by multiplex CRISPR/Cas9- based genome engineering system}

We newly developed a highly efficient multiplex CRISPR/Cas9-based genome engineering system for exon skipping by modifying the lentiGuide-Puro plasmid (Nat Methods), originally provided from Feng Zhang's laboratory ${ }^{18}$. We first designed single guide RNAs (sgRNAs) targeting intron 2 (sgRNA-in2) and intron 3 (sgRNA-in3) of human CTNNB1 gene by using the GPP sgRNA Designer web tool, and constructed U6-driven sgRNA-in2 and H1-driven sgRNA-in3 expression plasmids, respectively. After confirming the mutation efficiency of the two sgRNAs, we next generated a lentivirus vector for simultaneously expressing them (Fig. 1a), and infected it into the HuH7 cells constitutively expressing Streptococcus pyogenes Cas 9 nuclease (SpCas9). As expected, $C T N N B 1^{\Delta \mathrm{ex} 3}$ alleles were amplified by PCR of genomic DNA in the genetically engineered HuH7 cells ( $\left.\mathrm{HuH7-CTNNB1}{ }^{\triangle \mathrm{ex} 3}\right)$, and $\beta$-catenin ${ }^{\triangle A 5-A 80}$ proteins were detected in the pools of the HuH7-CTNNB1 ${ }^{\Delta \mathrm{ex} 3}$ cells (Fig. 1b,c). Similarly to the human HCC cells, mouse $\mathrm{HCC}$ cells expressing active form $\beta$-catenin were derived from the $3 \mathrm{H} 3$ cell line, which was a Hras $^{Q 61 L}$-mutated mouse HCC cell line isolated from the C57BL6/J MC4R-KO mouse model ${ }^{19}$, and then termed as $3 \mathrm{H} 3-\mathrm{Ctnnb}_{1}{ }^{\Delta \mathrm{ex} 3}$. Ctnnb ${ }^{\Delta \mathrm{ex} 3}$ alleles and $\beta$-catenin ${ }^{\triangle \mathrm{A} 5-\mathrm{A} 80}$ proteins were also identified at the DNA and protein levels in the $3 \mathrm{H} 3-\mathrm{Ctnnb} 1^{\triangle \mathrm{ex}} 3$ pools (Fig. $\left.1 \mathrm{~b}, \mathrm{C}\right)$. Thus, our multiplex CRISPR/Cas9-based genome engineering system could work efficiently for exon skipping. 


\section{Wnt/ $\beta$-catenin signaling activation in HCC cells with exon 3 skipping of $\beta$-catenin}

Subclones were established from the HuH7-CTNNB1 ${ }^{\Delta \mathrm{ex} 3}$ and $3 \mathrm{H} 3-\mathrm{Ctnnb} 1^{\Delta \mathrm{ex} 3}$ cell pools, and nuclear translocation of $\beta$-catenin ${ }^{\triangle A 5-A 80}$ proteins was enhanced in the HuH7-CTNNB1 ${ }^{\Delta \mathrm{ex} 3}$ and $3 \mathrm{H} 3-\mathrm{Ctnn} b 1^{\Delta \mathrm{ex} 3}$ subclones (Fig. 2a). To determine major downstream genes of the Wnt/ $\beta$-catenin signaling pathway in $\mathrm{HCC}$, we compared gene expression profiles between human HCC samples with and without mutations in exon 3 of $C T N N B 1$ by using public genome and transcriptome data sets provided from the Cancer Genome Atlas Research Network (TCGA) as shown in Supplementary Table 1. The differentially expressed genes included key components of the Wnt/ $\beta$-catenin signal transduction, such as $L G R 5$, RNF43, ZNRF3, AXIN2 and TCF7, implying positive and negative feedback loops. We examined the mRNA expression levels of them, and significant upregulation of LGR5, RNF43 and AXIN2 indicated activation of the $\beta$-catenin signaling in the HuH7-CTNNB1 $1^{\triangle \mathrm{ex} 3}$ and $3 \mathrm{H} 3-\mathrm{Ctnnb} 1^{\triangle \mathrm{ex} 3}$ subclones (Fig. 2b). Taken together, CRISPR/Cas9-mediated exon 3 skipping of $\beta$-catenin could molecularly and biologically mimic the $\beta$ catenin signaling activation in human and mouse HCC cells.

\section{Downregulation of immune-related gene sets by exon 3 skipping of $\beta$-catenin in HCC}

We performed RNA-seq analysis of the HuH7-CTNNB1 ${ }^{\triangle \mathrm{ex} 3}$ and $3 \mathrm{H} 3-\mathrm{Ctnnb} 1^{\triangle \mathrm{ex} 3}$ cells, and identified $L G R 5$, RNF43, AXIN2 and TMPRSS2 as commonly upregulated genes (log2 fold-change $>1.5$ and $P$-value $<10^{-}$ ${ }^{10}$ ), which was consistent with the results of quantitative RT-PCR analysis. Gene set enrichment analysis (GSEA) of the HuH7-CTNNB1 ${ }^{\Delta \mathrm{ex} 3}$ and $3 \mathrm{H} 3-\mathrm{Ctnnb} 1^{\mathrm{Aex} 3}$ cells revealed the close relationship between activation of the $\beta$-catenin signaling and downregulation of immune-associated gene sets (Fig. 3a). The HALLMARK TNFA SIGNALING VIA NFKB (M5890), GO HUMORAL IMMUNE RESPONSE (M13774) and GO REGULATION OF HUMORAL IMMUNE RESPONSE (M14968) gene sets were negatively enriched in both of the human and mouse HCC cells (Fig. 3b). These findings suggested that $\beta$-catenin signaling activation could contribute to immune evasion, and were consistent with previous studies of clinical specimens $7,13,16$.

\section{Immune evasion of mouse HCC cells with exon 3 skipping of $\beta$-catenin}

For evaluating inhibitory effects of the $\beta$-catenin signaling on immune surveillance, we investigated T cell killing of the $3 \mathrm{H} 3-\mathrm{Ctnnb} 1^{\triangle \mathrm{ex} 3}$ and control cells (3H3-Ctrl) as illustrated in Fig. 4a. Monocytes and T lymphocytes were obtained from C57BL6/J mice, and activated by conditioned media of each cell lines. By co-culture with immune cells, the number of the $3 \mathrm{H} 3-\mathrm{Ctrl}$ cells was notably decreased by more than 
$50 \%$, while the number of the $3 \mathrm{H} 3-\mathrm{Ctnnb} 1{ }^{\Delta \mathrm{ex} 3}$ cells was not changed (Fig. $\left.4 \mathrm{~b}\right)$. Three dimensional coculture system also showed the similar results (Fig. 4c).

\section{Downregulation of cytokine genes in HCC with $\beta$-catenin signaling activation}

The results of $T$ cell killing assays suggested that $\beta$-catenin signaling might control cytokines involved in immune cell activation. Among 114 cytokines registered in the CYTOKINE ACTIVITY gene set (M14581), 20 and 16 cytokines were remarkably downregulated in human and mouse $C T N N B 1^{\triangle \mathrm{ex} 3} \mathrm{HCC}$ cells, respectively, and CCL20, CSF1, CSF3, CXCL1, CXCL2, GDF15, NAMPT and VEGFA were commonly suppressed at the mRNA level (Fig. 5a), which was confirmed by quantitative RT-PCR analysis (Fig. 5b). We next compared the expression levels of the eight cytokine genes between HCC samples with and without mutations in exon 3 of CTNNB1 by using the TCGA data sets (Fig. 6), and identified significant downregulation of CCL2O (fold-change: 0.164 ; $P$-value: $1.58 \times 10^{-9}$ ), CXCL 1 (fold-change: 0.082 ; $P$-value: 9.65 $\times 10^{-15}$ ), CXCL2 (fold-change: 0.467; $P$-value: 0.002), NAMPT (fold-change: $0.521 ; P$-value: $5.04 \times 10^{-5}$ ) and VEGFA (fold-change: 0.927; $P$-value: 0.027).

\section{Discussion}

Although previous studies have examined the relationship between the $\mathrm{Wnt} / \mathrm{\beta}$-catenin signaling pathway and immune surveillance, they are artificial due to overexpression of mutated $\beta$-catenin, such as $\beta$ -

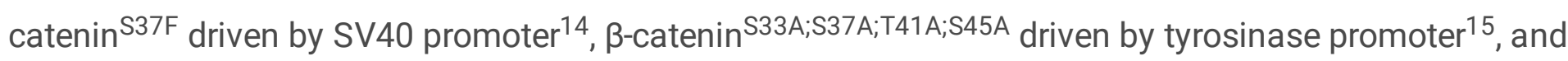
$\beta$-catenin ${ }^{\Delta N 90}$ driven by EF1a promoter ${ }^{17}$. To overcome this limitation, we first tried to knock in a mutated sequence of CTNNB1 exon 3 to human HCC cells with the help of the CRISPR/Cas9 system by using single strand DNA or plasmid donor, but it is difficult to obtain CTNNB1-mutated subclones because homology-directed repair is less dominant than non-homologous end joining (NHEJ) during double-strand break repair. We then mimicked activation of the $\beta$-catenin signaling by skipping exon 3 , that is, joining the ends of intron 2 and intron 3 simultaneously cleaved by the CRISPR/Cas 9 system. For this purpose, we established the novel multiplex CRISPR/Cas9-mediated genome engineering system of lentiCas9Blast and improved lentiGuide-Puro plasmids, although Kabadi et al. have already produced a lentivirus vector containing a Cas 9 transcription cassette and multiple sgRNA transcription cassettes ${ }^{20}$. This is because a Golden Gate cloning method is complicated compared with a conventional cloning method, and because two-vector system is superior to all-in-one vector system in functional viral titer ${ }^{18}$. Thus, our vector system enabled exon skipping with ease and efficiency, and could expand to other models, such as $E G F R^{\triangle \mathrm{ex} 19}$ and $E R B B 2^{\triangle \mathrm{ex} 16}$ for activation of the EGFR signaling pathway, and $P O L D 1^{\triangle \mathrm{ex} 10}$ and $P O L E^{\triangle \mathrm{ex} 9}$ for attenuation of exonuclease activity.

Immune checkpoint inhibitors (ICls) including anti-PD-1 and anti-PD-L1 antibodies have provided a revolutionary approach to cancer therapy, and clinical trials of ICls for various types of cancer are now 
ongoing and successful. In HCC, two anti-PD-1 antibodies nivolumab and pembrolizumab prolonged patient survival in phase II trials, however both monotherapies failed in phase III trials unfortunately. Harding et al. have revealed that CTNNB1-mutated HCC is more accumulated in the ICI-resistant group than in the ICl-sensitive group ${ }^{21}$, which is consistent with the important finding that CTNNB1 mutation is enriched in non-T cell-inflamed tumors insusceptible to ICI therapy ${ }^{13}$. Since the mutation rate of CTNNB1 gene is relatively higher in HCC than in other types of cancer (Supplementary Fig. 1), clinical trials of ICls should be conducted or subanalyzed for HCC with wild-type and mutated $\beta$-catenin separately. As described above, it is possible that the $\beta$-catenin signaling regulates not immune checkpoint molecules but cytokines for control of tumor immune microenvironment, such as upregulation of IL-10 $10^{14}$ and downregulation of CCL4 ${ }^{15}$. Ruiz de Galarreta et al. demonstrated that antigen-expressing MYC,Trp53-/HCC evaded the immune system by decreasing CCL5 expression through activation of the $\beta$-catenin signaling pathway, and that CCL5 overexpression restored immunosurveillance in antigen-expressing $M Y C, C T N N B 1^{\triangle N 90} \mathrm{HCC}^{17}$. In contrast, this study demonstrated that endogenous active form $\beta$-catenin downregulated immune-associated signaling pathways in both human and mouse HCC by bioinformatic analysis, and that tumor-intrinsic $\beta$-catenin activation suppressed T cell cytotoxicity through cytokine secretion by in vitro assays.

By comparing the present and previous studies ${ }^{17}$, CCL20 and CXCL1 were commonly downregulated in HCC with $\beta$-catenin signaling activation. CCL20, alternatively named liver and activation-regulated chemokine (LARC), was originally discovered in the liver and strongly expressed in mononuclear cells near necrosis in the chronically inflamed liver and HCC. CCR6 is the selective receptor for CCL20, and the CCR6-CCL20 axis contributes to the recruitment of immature DC to the antigen entry site and the arrest of $\mathrm{T}$ lymphocyte on the endothelium in the early phase of immune response ${ }^{22}$. The chemokine receptor CXCR2 and its ligands CXCL1, CXCL2, CXCL3, CXCL5 and CXCL8 play critical roles in the chemoattraction of neutrophils towards tumor tissues. Similarly to tumor-associated macrophages, tumor-associated neutrophils can be polarized into either an antitumoral (N1) or a protumoral (N2) phenotype; the $\mathrm{N} 1$ phenotype is induced by TGF- $\beta$ blockade, and expresses immunoactivating cytokines and chemokines for killing cancer cells ${ }^{23}$. Thus, the $\beta$-catenin signaling pathway might suppress immune response through decrease of cytokine levels.

In conclusion, this study enabled intrinsic $\beta$-catenin signaling activation by developing the highly efficient CRISPR/Cas9-based exon skipping system, and showed that it could contribute to immune evasion by suppressing immunoactivating cytokines including CCL20 and CXCL1. The CTNNB1-mutated HCC subtype accounts for approximately $30 \%$ of all cases (Supplementary Fig. 1), but is refractory to ICI therapy. Since clinical trials evaluating recombinant cytokines as immunostimulants in cancer patients have recently been launched ${ }^{24}$, transarterial infusion of the candidate immunoactivating cytokines could also be effective to the subtype.

\section{Methods}




\section{Ethics statement}

The study was carried out in compliance with the ARRIVE guidelines. All methods were performed in accordance with relevant guidelines and regulations. All experimental protocols were approved by Institutional Review Board (G2018-132C5, Medical Research Ethics Committee for Genetic Research of Tokyo Medical and Dental University; A2019-263C2, Institutional Animal Care and Use Committee of Tokyo Medical and Dental University).

\section{Cell culture}

Human HCC cell line HuH7 was purchased from the American Type Culture Collection (Manassas, VA). Mouse cell line $3 \mathrm{H} 3$ was derived from HCC tumor grown in a C57BL/6J MC4R-KO mouse fed with high fat $\operatorname{diet}^{19}$. They were cultured in RPMI-1640 and DMEM (Wako, Osaka, Japan) medium containing 10\% fetal bovine serum (FBS), and 1\% penicillin, streptomycin and amphotericin B (Wako), maintained in a humidified incubator at $37{ }^{\circ} \mathrm{C}$ in $5 \% \mathrm{CO} 2$, and harvested with $0.05 \%$ trypsin-0.03\% EDTA (Wako).

\section{Exon 3 skipping of $\beta$-catenin by multiplex CRISPR/Cas9- based genome engineering system}

To generate the backbone plasmid for the CRISPR/Cas9 system, the lentiGuide-Puro (Addgene \#52963) was modified by inserting a $K p n$ site in front of the $U 6$ promoter and replacing the Hindlll site behind the sgRNA scaffold with an EcoRI site, named as LG-U6. The LG-H1 plasmid was also produced by replacing the U6 promoter with the $\mathrm{H} 1$ promoter in the LG-U6 plasmid. The LG-U6 and LG-H1 plasmid for expressing sgRNAs targeting intron 2 and intron 3 of $\beta$-catenin (sgRNA-in2 and sgRNA-in3) were constructed following the manufacture's manual (Supplementary Table 2). The H1-sgRNA-in3 sequence was tandemly cloned into the EcoRI site of the LG-U6-sgRNA-in2 plasmid (Figure 1A). The HuH7 and 3H3 cells were sequentially infected with the lentiviral vectors for constitutively expressing SpCas9 (lentiCas9-Blast; Addgene \#52962) and simultaneously expressing sgRNA-in2 and sgRNA-in3, and then treated with 10 $\mu \mathrm{g} / \mathrm{mL}$ blasticidin and $10 \mu \mathrm{g} / \mathrm{mL}$ puromycin, respectively. The subclones with $\beta$-catenin alleles lacking exon 3 were isolated by limiting dilution.

\section{DNA extraction and PCR analysis}

Cell pellets were suspended in TNE Buffer (10 mM Tris- $\mathrm{HCl}$, pH 8.0; 150 mM NaCl; 2 mM EDTA; 0.5\% SDS) with $1 \%$ proteinase $\mathrm{K}$ (TaKaRa Bio, Shiga, Japan) at $55^{\circ} \mathrm{C}$ overnight. Genomic DNA was obtained from cells by phenol-chloroform extraction. The primer sets and amplification conditions for PCR are listed in Supplementary Table 3. 


\section{RNA extraction}

Total RNA was extracted from cells by using RNeasy Plus Mini Kit (QIAGEN, Germantown, MD). Contaminating DNA was removed by digestion with RNase-Free DNase Set (QIAGEN).

\section{Quantitative RT-PCR analysis}

For single-stranded complementary DNA synthesis, $1 \mu \mathrm{g}$ of total RNA was reverse-transcribed by SuperScript III Reverse Transcriptase (Thermo Fisher Scientific, Waltham, MA). Quantitative RT-PCR analysis was performed by using TB Green Premix Ex Taq II (TaKaRa Bio) with StepOne real-time PCR system (Thermo Fisher Scientific) according to the manufacturer's instructions, and the $\Delta \Delta \mathrm{Ct}$ method was used for quantification. GAPDH was used as an internal control. The primer sets and amplification conditions for PCR are listed in Supplementary Table 4.

\section{RNA sequencing analysis}

Sequencing libraries were prepared from total RNA with the TruSeq Standard mRNA Library Kit (Illumina, San Diego, CA), and RNA sequencing was run on an Illumina NovaSeq 6000 . Sequence reads were aligned to the human and mouse reference genome (GRCh38 and GRCm38) by STAR (2.7.0d), and transcript quantification was performed by RSEM (1.3.1). Differentially expressed genes were determined by using DESeq2 (1.14.1).

\section{Western Blotting}

After whole cell lysates were collected by using ice-cold RIPA buffer (Thermo Fisher Scientific), $30 \mu \mathrm{g}$ of protein from each sample was subjected to electrophoresis through $10 \%$ sodium dodecyl sulfatepolyacrylamide gels and transferred onto Immobilon polyvinyldifluoride membranes (Millipore, Bedford, MA). The membrane was blocked with $5 \%$ skimmed milk or bovine serum albumin for an hour at room temperature, and then incubated overnight at $4{ }^{\circ} \mathrm{C}$ with primary antibodies as follows; $\beta$-catenin (D10A8, 1:1000), GAPDH (14C10, 1:1000) and lamin B1 (D6V6H, 1:1000), all of which were purchased from Cell Signaling Technology (Danvers, MA). Secondary antibodies were added, and signals were detected by using Clarity Western ECL Substrate (Bio-Rad, Hercules, CA) with LAS-3000 (Fujifilm, Tokyo, Japan).

\section{Subcellular fractionation analysis}

Cytoplasmic and nuclear proteins were separately extracted by NE-PER Nuclear and Cytoplasmic Extraction Reagents (Thermo Fisher Scientific) according to the manufacturer's instructions, and then Western blotting analysis was performed. GAPDH and lamin B1 were used for detecting cytoplasmic and nuclear fractionated protein, respectively. 


\section{Isolation of T cells}

Eight-week-old male C57BL/6J mice were euthanized, and spleens were resected and disrupted with a flat plunger tip of a $5 \mathrm{~mL}$ syringe. After hemolysis, whole splenocytes were incubated in a nylon wool fiber column to remove $B$ lymphocytes for an hour at $37^{\circ} \mathrm{C}$. T lymphocytes were collected and cultured in RPMI-1640 medium supplemented with 10\% FBS, 1\% ITS supplement (Thermo Fisher Scientific), 100 $\mathrm{U} / \mathrm{ml}$ murine IL-2 (Peprotech, Cranbury, NJ) and $10 \mathrm{ng} / \mathrm{ml}$ murine IL-7 (Peprotech).

\section{Immune-cell preparation}

Isolation of mouse bone marrow and differentiation of DCs was performed as previously described ${ }^{25}$. Briefly, eight-week-old male C57BL/6J mice were euthanized, and bone marrow was flushed out from femur and tibia by using a $1 \mathrm{ml}$ syringe and a $27 \mathrm{G}$ needle. Bone marrow-derived monocytes (BMDMs) were washed, and then cultured in DC differentiation medium as follows; RPMI-1640, 10\% FBS, $1 \%$ penicillin-streptomycin-amphotericin B, $20 \mathrm{ng} / \mathrm{ml}$ murine GM-CSF (Peprotech) and $5 \mathrm{ng} / \mathrm{ml}$ murine IL-4 (Peprotech). Six days after preculture, differentiated bone marrow-derived dendritic cells (BMDCs) were further cultured in conditioned medium collected from the $3 \mathrm{H} 3-\mathrm{Ctrl}$ cells or $3 \mathrm{H} 3-\mathrm{CTNNB} 1^{\mathrm{Dex} 3}$ cells for 24 hours to stimulate with cancer antigens.

\section{T cell killing assay}

A day after T lymphocytes were co-cultured with BMDCs for priming, the $3 \mathrm{H} 3$ cells or the $3 \mathrm{H} 3-\mathrm{Ctnnb} 1{ }^{\Delta \mathrm{ex} 3}$ cells were plated at $5 \times 10^{3}$ cells per well in a 24 -well tissue culture plate (for two-dimensional culture) or ultra-low attachment plate (for sphere culture) with primed immune cells for 48 hours. Advanced DMEM/F12 (Thermo Fisher Scientific) with 0.5\% B-27 Supplement (Thermo Fisher Scientific), $20 \mathrm{ng} / \mathrm{ml}$ human EGF (Peprotech) and $1 \mu \mathrm{g} / \mathrm{ml}$ human FGF-basic (Peprotech) was used for sphere formation. To evaluate cytotoxic activity of immune cells in two-dimensional culture, cell viability was estimated by using CellTiter-Glo 2.0 reagent (Promega, Madison, WI) with FLUOstar OPTIMA-6 microplate reader (BMG Labtech, Durham, NC) according to the manufacturer's instructions. For sphere culture, cancer cell area was measured by using ImageJ software.

\section{Bioinformatic analysis}

Gene set enrichment analysis was performed with the MSigDB gene sets. Public genome and transcriptome data of $373 \mathrm{HCC}$ samples were provided from the Cancer Genome Atlas Research Network, and downloaded from the cBioPortal site. Genome data divided them into 77 and 296 tumors with and without CTNNB1 hotspot mutations.

\section{Declarations}




\section{Acknowledgements}

We gratefully thank Prof. Takashi Yamamoto and Prof. Tetsushi Sakuma (Department of Mathematical and Life Sciences, Graduate School of Science, Hiroshima University) for supporting the establishment of multiplex CRISPR/Cas9 system. We also thank Ms. Hiromi Nagasaki for technical assistance. This work was supported by Grants-in-Aid for Scientific Research (A; 19H01055) and Challenging Research (Exploratory; 20K21627) from the Ministry of Education, Culture, Sports, Science and Technology of Japan; Research Grant from the Princess Takamatsu Cancer Research Fund; P-CREATE (JP19cm0106540), and Program for Basic and Clinical Research on Hepatitis (JP20fk0210040, JP20fk0210060) from AMED (Japan Agency for Medical Research and Development).

\section{Author contributions}

M.A., S.S., A.Kabashima, Y.A. and M.S. performed the experiments. M.A., S.S., and A.Kabashima analyzed the data. S.S performed bioinformatics. S.S. and S.Y. supervised the construction of lentivirus vectors. M.A., S.S., and A.Kabashima wrote the manuscript with comments from all authors. K.A., A.Kudo, and M.T. elaborated the manuscript. S.T. conceptualized, designed and supervised the study.

\section{Competing interests}

The authors declare no competing interests.

\section{References}

1. Yang, J. D. et al.A global view of hepatocellular carcinoma: trends, risk, prevention and management. Rev. Gastroenterol. Hepatol. 16, 589-604 (2019).

2. Finn, R. S. et al. Atezolizumab plus bevacizumab in unresectable hepatocellular carcinoma. Engl. J. Med. 382, 1894-1905 (2020).

3. Llovet, J. M., Montal, R., Sia, D. \& Finn, R. S. Molecular therapies and precision medicine for hepatocellular carcinoma. Rev. Clin. Oncol.15, 599-616 (2018).

4. Kumar-Sinha, C. \& Chinnaiyan, A. M. Precision oncology in the age of integrative genomics. Biotechnol.36, 46-60 (2018).

5. Llovet, J. M., Villanueva, A., Lachenmayer, A. \& Finn, R. S. Advances in targeted therapies for hepatocellular carcinoma in the genomic era. Rev. Clin. Oncol.12, 408-424 (2015).

6. Zucman-Rossi, J., Villanueva, A., Nault, J. \& Llovet, J. M. Genetic landscape and biomarkers of hepatocellular carcinoma. Gastroenterology149, 1226-1239 (2015).

7. Shimada, S. et al.Comprehensive molecular and immunological characterization of hepatocellular carcinoma. EBioMedicine 40, 457-470 (2019). 
8. de La Coste, A. et al.Somatic mutations of the beta-catenin gene are frequent in mouse and human hepatocellular carcinomas. Natl. Acad. Sci. USA 95, 8847-8851 (1998).

9. Miyoshi, Y. et al.Activation of the beta-catenin gene in primary hepatocellular carcinomas by somatic alterations involving exon 3. Cancer Res. 58, 2524-2527 (1998).

10. Delmas, V. et al.Beta-catenin induces immortalization of melanocytes by suppressing p 16 INK4a expression and cooperates with N-Ras in melanoma development. Genes Dev. 21, 2923-2935 (2007).

11. Tward, A. D. et al.Distinct pathways of genomic progression to benign and malignant tumors of the liver. Natl. Acad. Sci. USA 104, 14771-14776 (2007).

12. Harada, N. et al.Intestinal polyposis in mice with a dominant stable mutation of the beta-catenin gene. EMBO J. 18, 5931-5942 (1999).

13. Luke, J. J., Bao, R., Sweis, R. F., Spranger, S. \& Gajewski, T. F. WNT/ß-catenin pathway activation correlates with immune exclusion across human cancers. Cancer Res.25, 3074-3083 (2019).

14. Yaguchi, T. et al.Immune suppression and resistance mediated by constitutive activation of Wnt/ $\beta$ catenin signaling in human melanoma cells. Immunol. 189, 2110-2117 (2012).

15. Spranger, S., Bao, R. \& Gajewski, T. F. Melanoma-intrinsic $\beta$-catenin signalling prevents anti-tumour immunity. Nature523, 231-235 (2015).

16. Sia, D. et al.Identification of an immune-specific class of hepatocellular carcinoma, based on molecular features. Gastroenterology 153, 812-826 (2017).

17. Ruiz de Galarreta, M. et al. $\beta$-Catenin activation promotes immune escape and resistance to anti-PD-1 therapy in hepatocellular carcinoma. Cancer Discov. 9, 1124-1141 (2019).

18. Sanjana, N. E., Shalem, O. \& Zhang, F. Improved vectors and genome-wide libraries for CRISPR screening. Methods11, 783-784 (2014).

19. Itoh, M. et al.Melanocortin 4 receptor-deficient mice as a novel mouse model of nonalcoholic steatohepatitis. J. Pathol. 179, 2454-2463 (2011).

20. Kabadi, A. M., Ousterout, D. G., Hilton, I. B. \& Gersbach, C. A. Multiplex CRISPR/Cas9-based genome engineering from a single lentiviral vector. Nucleic Acids Res.42, e147 (2014).

21. Harding, J. J. et al.Prospective genotyping of hepatocellular carcinoma: clinical implications of nextgeneration sequencing for matching patients to targeted and immune therapies. Cancer Res. 25, 2116-2126 (2019).

22. Schutyser, E., Struyf, S. \& Damme, J. V. The CC chemokine CCL20 and its receptor CCR6. Cytokine Growth Factor Rev.14, 409-426 (2003).

23. Powell, D. R. \& Huttenlocher, A. Neutrophils in the tumor microenvironment. Trends Immunol.37, 4152 (2016).

24. Berraondo, P. et al.Cytokines in clinical cancer immunotherapy. J. Cancer 120, 6-15 (2019).

25. Madaan, A., Verma, R., Singh, A. T., Jain, S. K. \& Jaggi, M. A stepwise procedure for isolation of murine bone marrow and generation of dendritic cells. Biol. Methods 1, e1 (2014). 


\section{Figures}

Figure 1

a

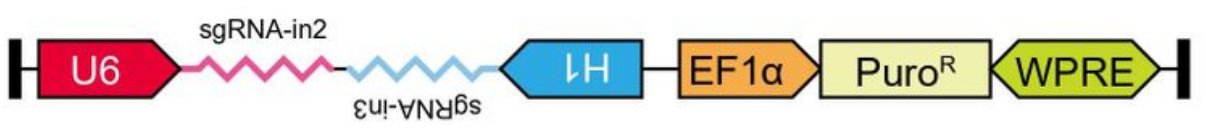

\begin{tabular}{|l|l|l|l|l|l|l|}
\hline EF1a & SpCas9 & $2 \mathrm{~A}$ & Blast $^{\mathrm{W}}$ & WPRE \\
\hline
\end{tabular}

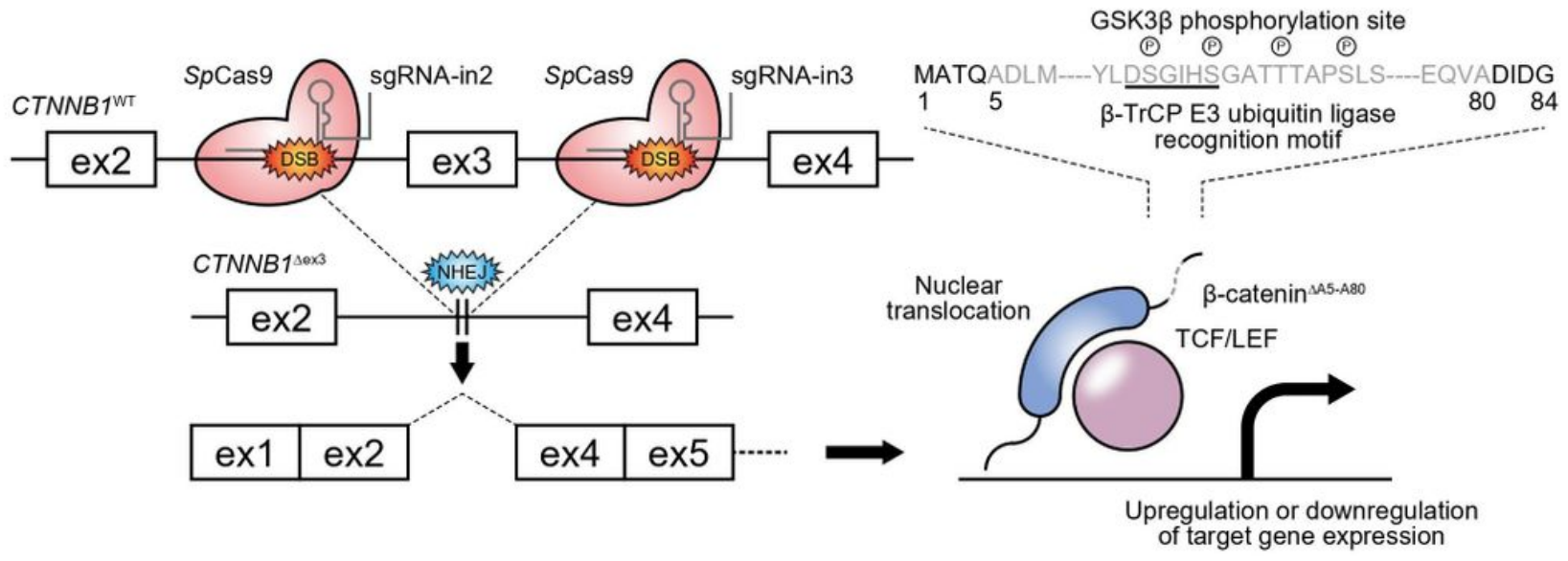

b

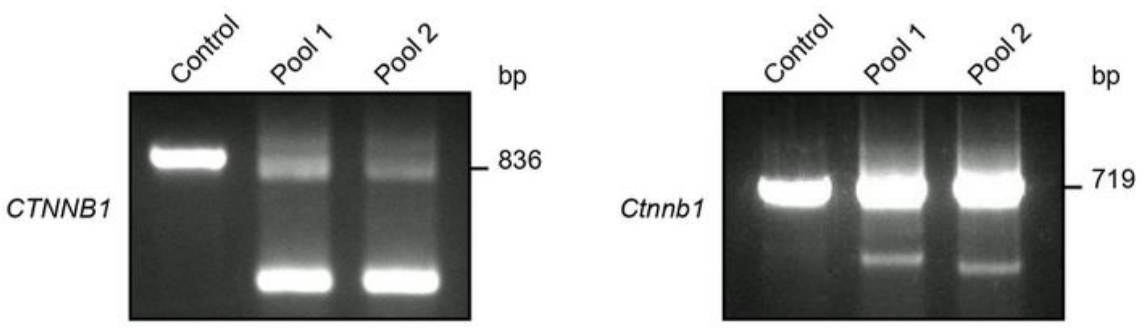

C

$\mathrm{HuH7}$

$3 \mathrm{H} 3$

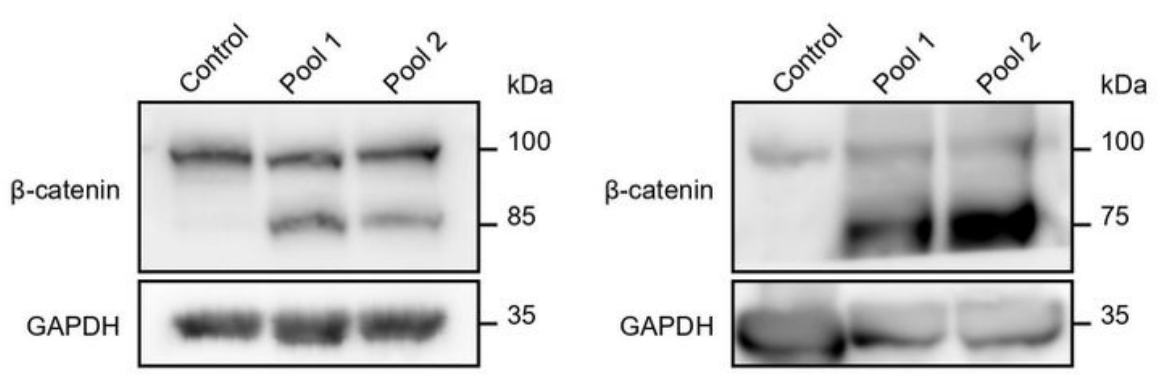

\section{Figure 1}

CRISPR/Cas9-mediated exon 3 skipping of $\beta$-catenin. (a) Schematic representation of exon 3 skipping of $\beta$-catenin (CTNNB1). (b) and (c) PCR and immunoblot analysis of $\beta$-catenin in human and mouse HCC cell lines, $\mathrm{HuH7}$ and $3 \mathrm{H} 3$. The expected PCR product sizes of wild-type and exon 3-skipping $\beta$-catenin are 836 bp, 294 bp (pool 1) and 294 bp (pool 2) in humans and 719 bp, 307 bp (pool 1) and 280 bp (pool 2) 
in mice, respectively. The molecular weights of $\beta$-cateninwild-type and $\beta$-catenin $\triangle A 5-A 80$ are $85 \mathrm{kDa}$ and $77 \mathrm{kDa}$ in both humans and in mice. GAPDH was used as a loading control.

\section{Figure 2}

a

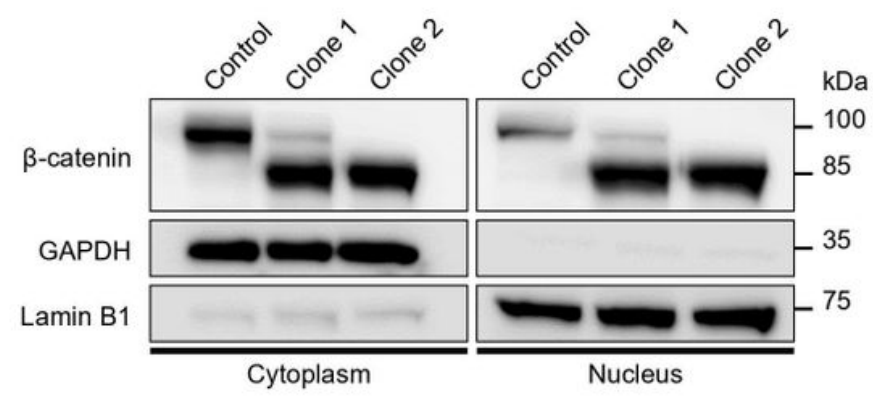

$\mathrm{HuH} 7$

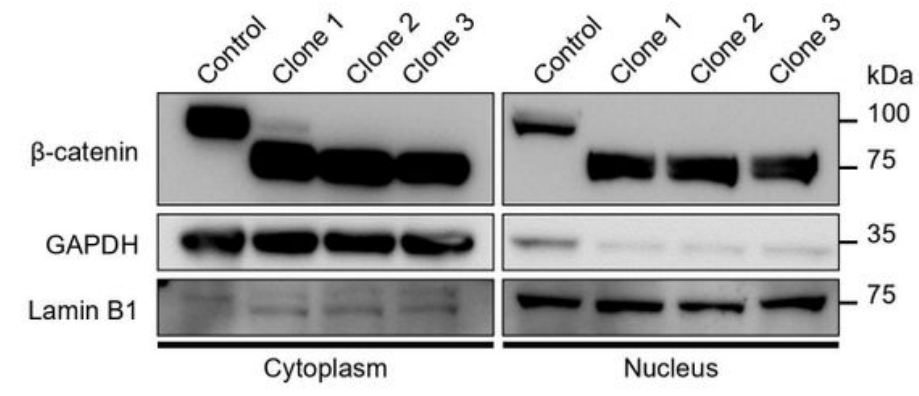

b

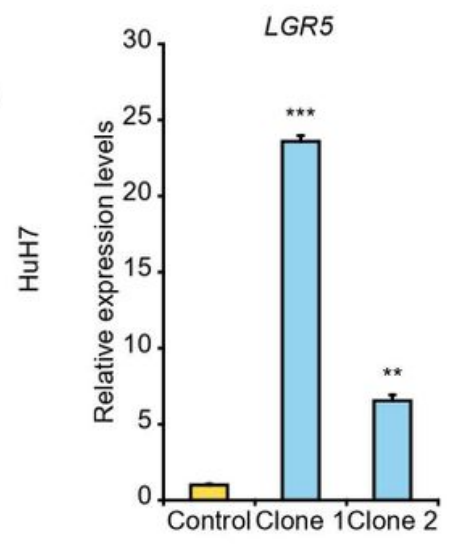

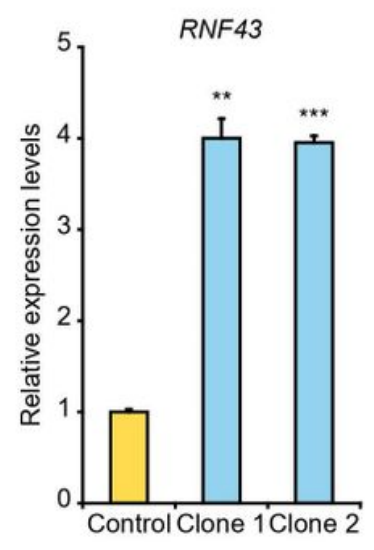
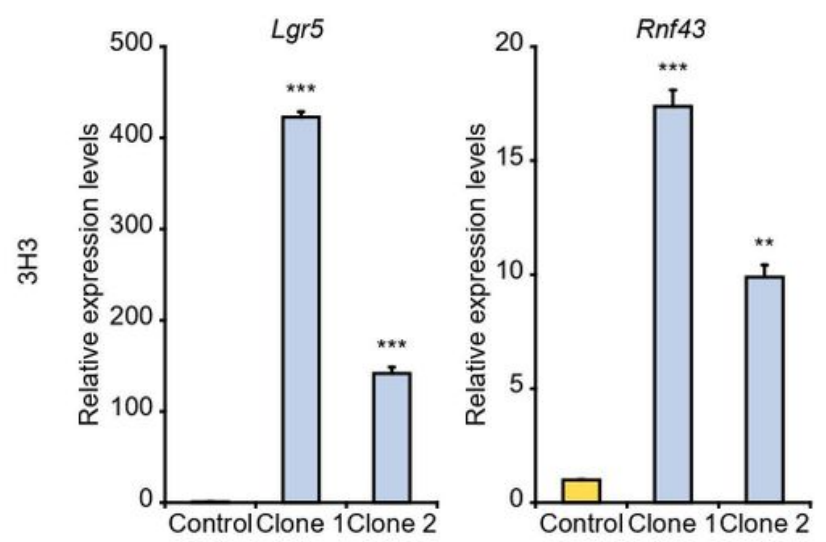
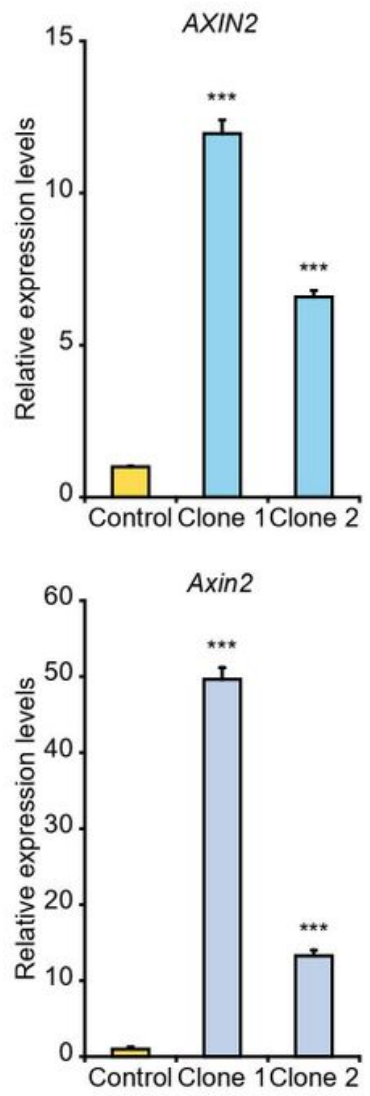

\section{Figure 2}

Activation of the Wnt/ $\beta$-catenin signaling pathway in HCC cells harboring exon 3 skipping $\beta$-catenin. (a) Immunoblot analysis of cytoplasmic and nuclear protein fractions of the HuH7-CTNNB1 $\triangle$ ex3 and $3 \mathrm{H} 3-$ Ctnnb1 $\triangle$ ex3 cells. GAPDH and lamin B1 were used as controls of the cytoplasmic and nuclear protein fractions, respectively. (b) Quantitative RT-PCR analysis of downstream genes of the Wnt/ $\beta$-catenin signaling pathway. Error bars are the mean $\pm \mathrm{SD}$. P-values were calculated by Welch's t-test. ${ }^{*} \mathrm{P}<0.01$; $\star \star \star P P 0.001$. 
Figure 3

a
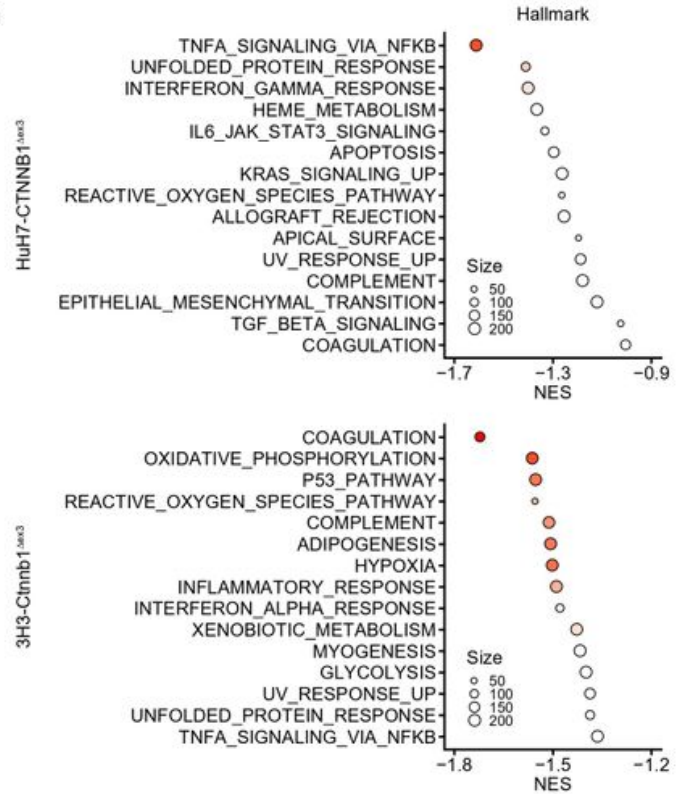
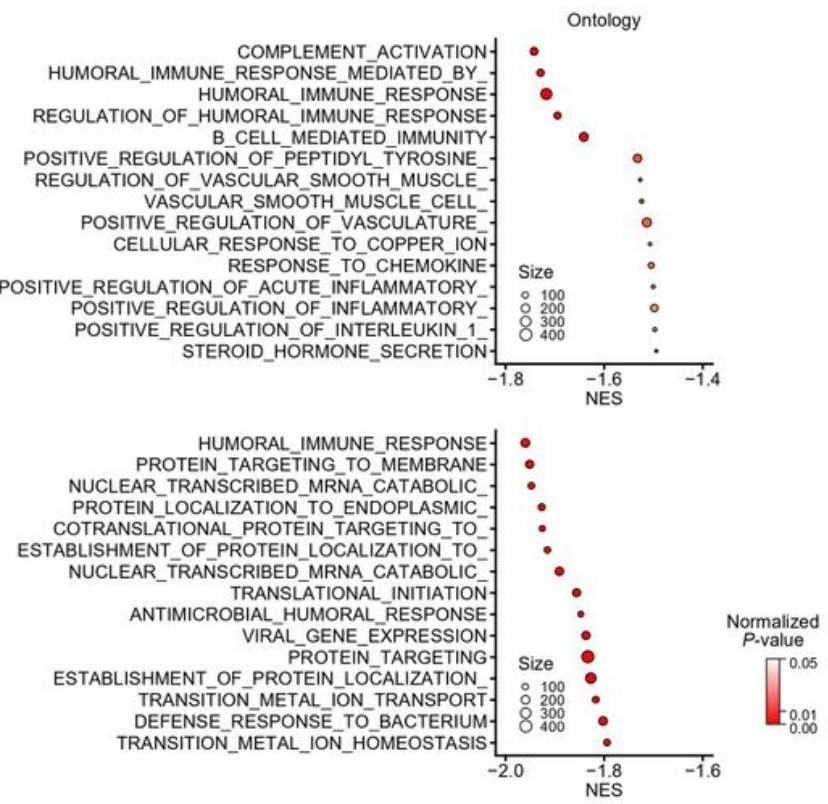

b

HALLMARK TNFA SIGNALING_

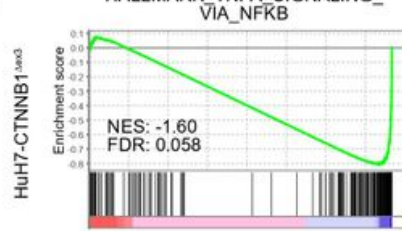

GO_HUMORAL_IMMUNE_RESPONSE

GO REGULATION_OF
MORAL_IMMUNE_RESPŌNSE
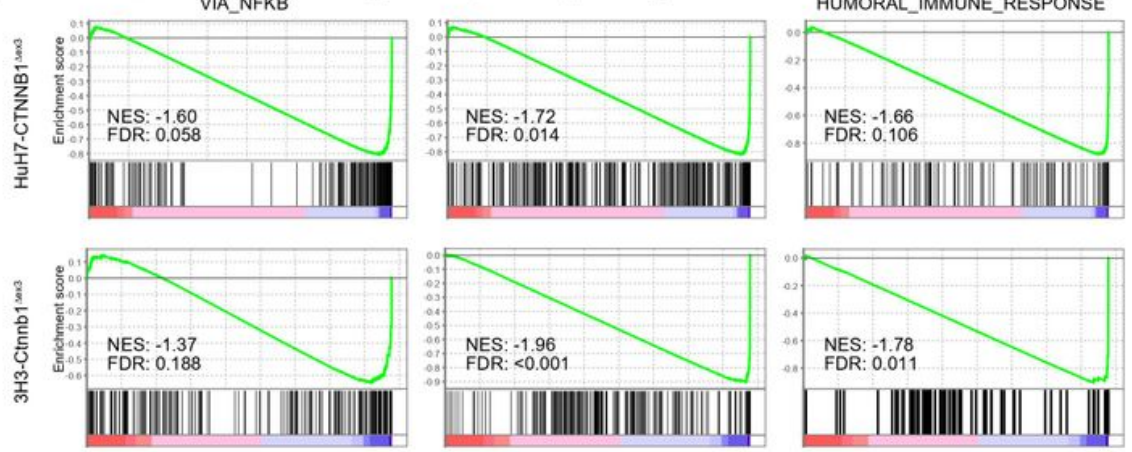

\section{Figure 3}

Alteration of signaling pathways by exon 3 skipping of $\beta$-catenin in HCC. (a) Bubble plots of gene sets negatively enriched in the HuH7-CTNNB1 $\triangle \mathrm{ex} 3$ and $3 \mathrm{H} 3-\mathrm{Ctnnb} 1 \Delta \mathrm{ex} 3$ cells. (b) Enrichment plots of gene sets commonly associated with the HuH7-CTNNB1 $\triangle$ ex3 and 3H3-Ctnnb1 $\Delta$ ex3 cells. Hallmark $(\mathrm{H}$ : hallmark) and ontology (C5.BP: gene ontology biological process) gene sets were obtained from the MSigDB. Normalized enrichment score (NES), normalized P-value and false discovery rate (FDR) were calculated by the GSEA application. 
Figure 4

a

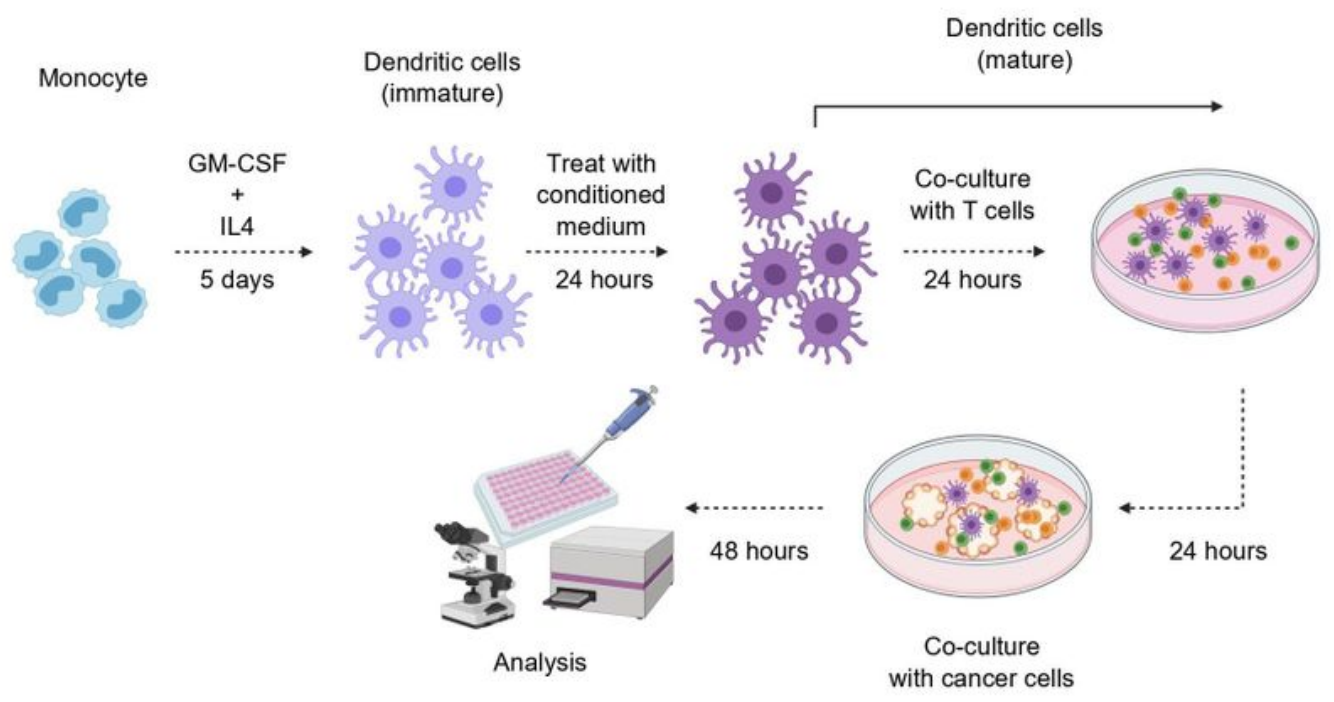

b
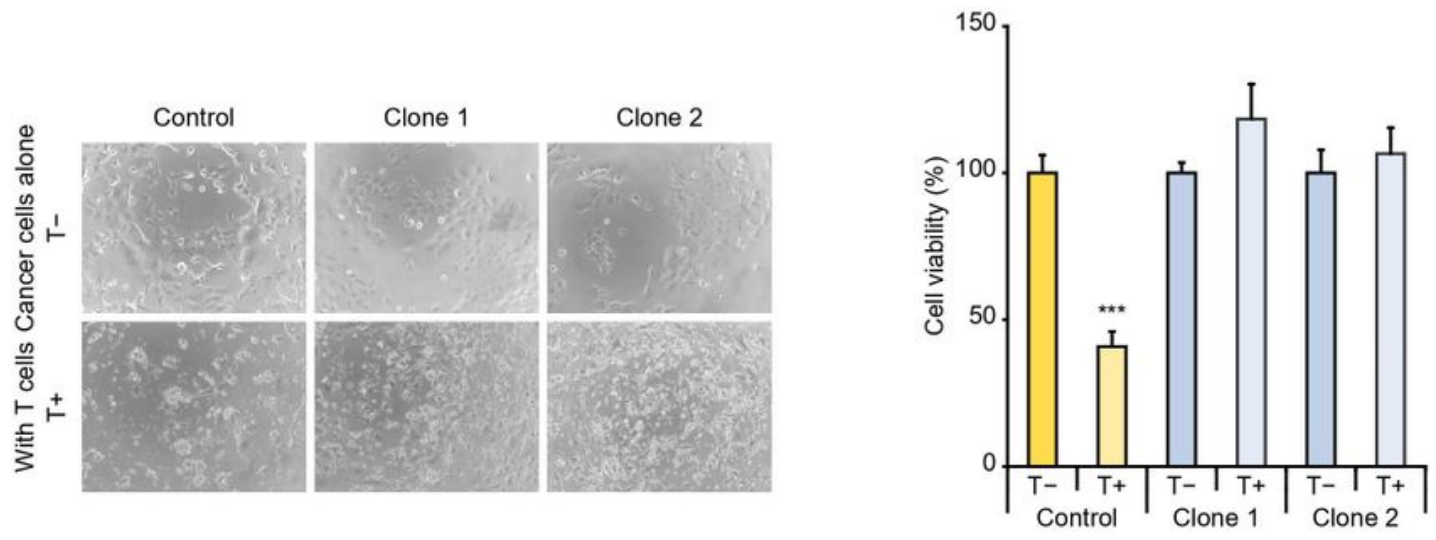

C
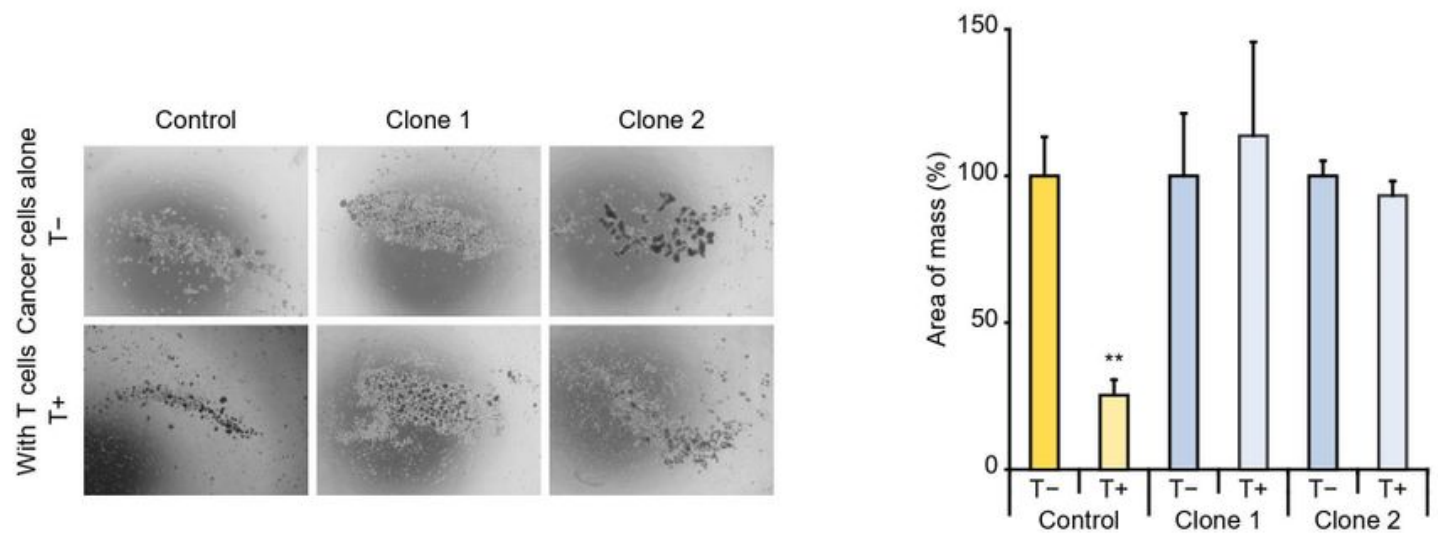

\section{Figure 4}

Immune evasion of mouse HCC cells with $\beta$-catenin signaling activation. (a) Schematic representation of in vitro T cell killing assays. (b) and (c) Two- and three-dimensional T cell killing assays of the 3H3Ctnnb1 1 ex3 cells. The left and right panels show representative phase-contrast images and cell viability data, respectively. Error bars are the mean \pm SD. P-values were calculated by Welch's t-test. **P $<0.01$;

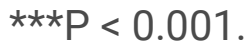


Figure 5

a
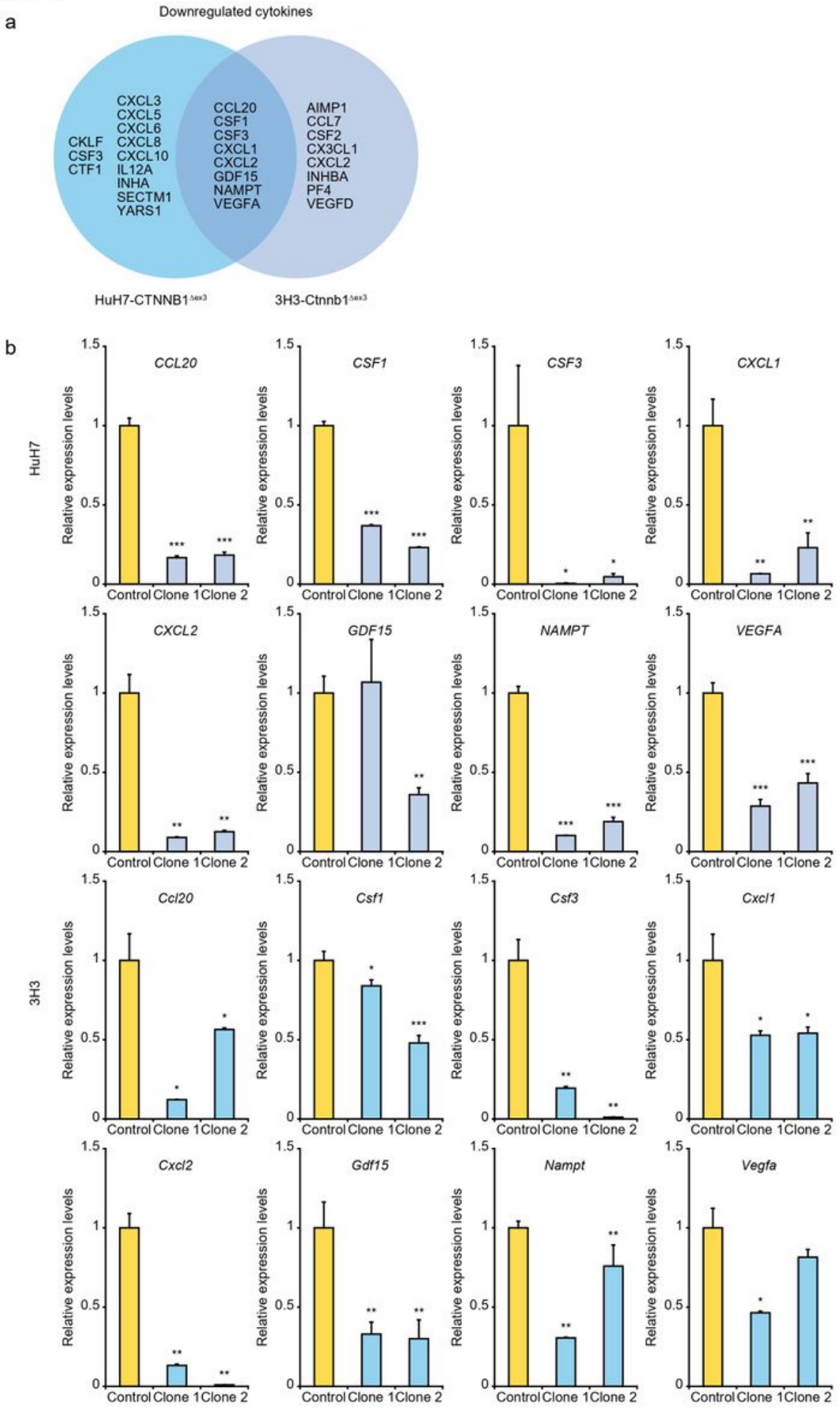

\section{Figure 5}

Downregulation of cytokines in human and mouse HCC cells with exon 3 skipping of $\beta$-catenin. (a) Venn diagram of cytokine genes downregulated in the HuH7-CTNNB1 $\Delta$ ex3 and $3 H 3-C t n n b 1 \Delta$ ex3 cells. Twenty and sixteen genes were extracted from 114 genes registered in the CYTOKINE ACTIVITY gene set (foldchange $<0.66$ and P-value $<0.05$ ). (b) Quantitative PCR analysis of eight candidate cytokine genes 
downregulated by $\beta$-catenin signaling activation. Error bars are the mean \pm SD. P-values were calculated by Welch's t-test. ${ }^{*} \mathrm{P}<0.05 ;{ }^{* \star} \mathrm{P}<0.01 ; * \star \star \mathrm{P}<0.001$.

Figure 6
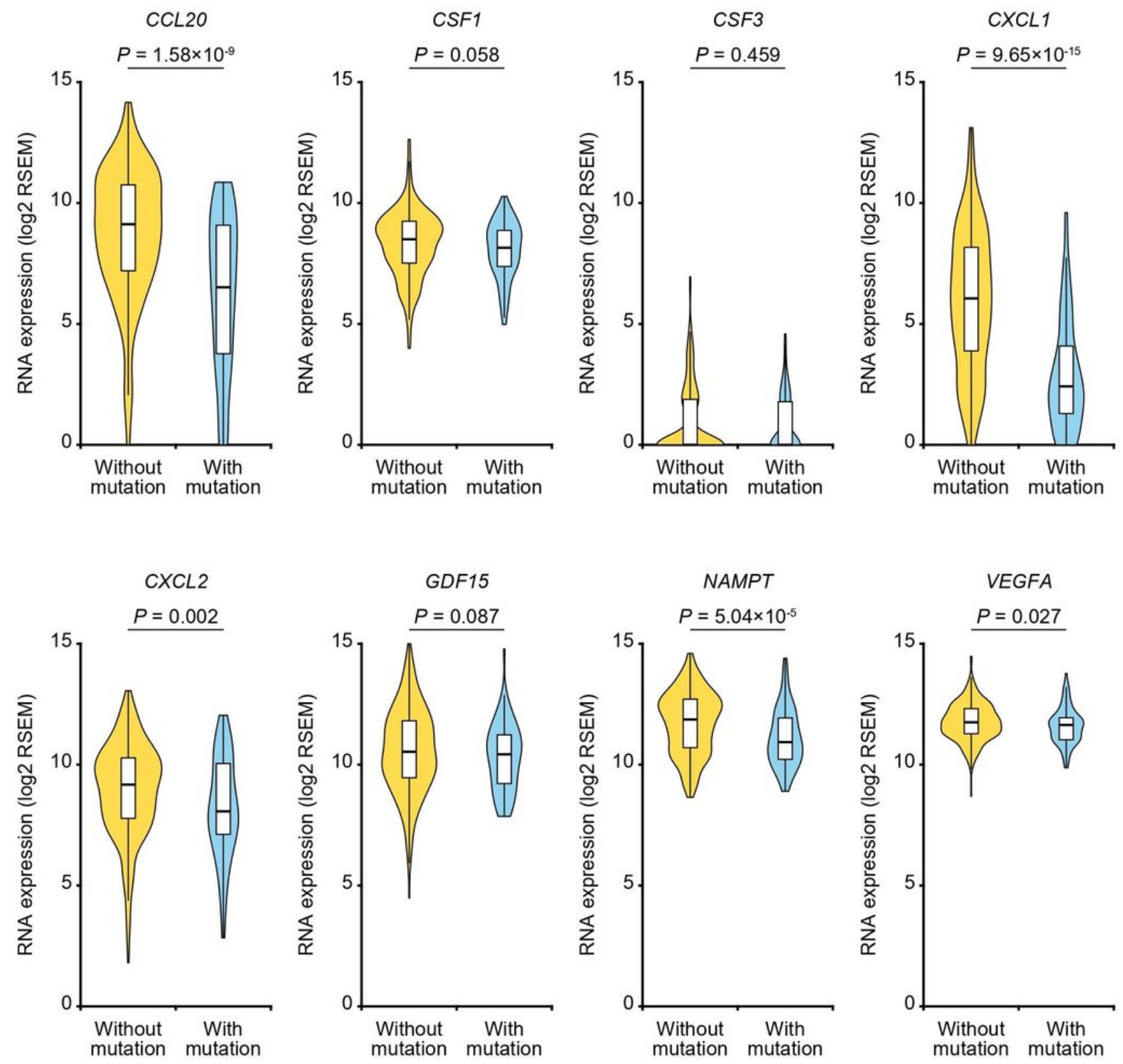

Figure 6

Downregulation of cytokines in human HCC samples with CTNNB1 hotspot mutations. Boxes in violin plots represent the interquartile range (range from the 25th to the 75th percentile), and horizontal lines show the median values. P-values were calculated by Mann-Whitney $\mathrm{U}$ test.

\section{Supplementary Files}


This is a list of supplementary files associated with this preprint. Click to download.

- SupplementaryFigure1.pdf

- supplementarytables.xlsx 